\title{
THE STATISTICS OF LOW-LEVEL COUNTING USING THE NEW GENERATION OF PACKARD LIQUID SCINTILLATION COUNTERS
}

\author{
G. T. COOK ${ }^{1}$ E. M. SCOTT ${ }^{2}$, E. M. WRIGHT ${ }^{2}$ and ROBERT ANDERSON ${ }^{1}$
}

\begin{abstract}
We consider the suitability of commonly used Poisson counting statistics applied to background count rates measured in the new generation of low-background Packard liquid scintillation spectrometers. We also investigate the stability of these systems over long time intervals. Undetected instability will result in an underestimation of the precision of any result (i.e., the calculated error will be too small), and, in the presence of a systematic source, could lead to inaccurate results. The work described here forms only a small part of a project to investigate the statistical criteria that should be applied to the performance of such counters. The procedures to be discussed here include the Poisson index of dispersion, $\bar{x}$ and $\mathbf{s}$ control charts and the MSSD test for detection of drift. These are illustrated on background count rates derived from the Packard 2260XL and 2000CA/LL.
\end{abstract}

\section{INTRODUCTION}

The current generation of liquid scintillation counters, e.g., LKB-Pharmacia Quantulus, Packard 2000 series counters employing burst-counting circuitry and others, offers considerable reductions in background count rates. The burst-counting circuitry technique used in the Packard instruments enables background count-rate reductions to be achieved through the examination of individual pulse shapes. Non-quenchable background events generally have a fast prompt pulse followed by a burst of after-pulses, whereas true $\beta$-events have fewer or no after-pulses.

Noakes \& Valenta (1989) take advantage of this difference in pulse shape to remove most of this unquenchable component of background. This enables both smaller and older samples to be dated with improved precision. It is important to establish that these count rates are stable, because excess variability will undoubtedly lead to reduced precision and possible measurement inaccuracies. Similarly, it is important to establish the suitability of Poisson statistics for such low count rates. We have considered results from both the Packard 2000CA/LL and Packard 2260XL counters for different counting geometries to: 1) investigate the appropriateness of Poisson counting statistics; 2) estimate, where required, excess variability in the data; 3 ) establish the background stability of these counting systems. We concentrate here on characterizing the background behavior.

We studied 12 background samples (both commercially purchased scintillation-grade benzene and benzene synthesized from 'infinite-age' material). Sample counting in this laboratory follows the quasi-simultaneous batch-counting technique. Each vial undergoes 50-min counts.

The 12 samples comprise 3 groups of 4 (Table 1) as follows:

1. $2 \mathrm{~g}$ benzene $+0.42 \mathrm{~g}$ of a toluene-based cocktail containing 12 and $6 \mathrm{~g} \mathrm{liter}^{-1}$ of butylPBD and bis-MSB, respectively, counted in a Packard 2000CA/LL; we made 100 measurements in 4 months.

2. $2 \mathrm{~g}$ of benzene $+6 \mathrm{mg}$ of both butyl-PBD and bis-MSB counted in a Packard 2260XL; we made 100 measurements in 4 months.

3. $0.5 \mathrm{~g}$ benzene $+1.5 \mathrm{mg}$ of both butyl-PBD and bis-MSB counted in a Packard 2260XL; we made 50 measurements in 1 month.

Figures $1 \mathrm{~A}$ and $1 \mathrm{~B}$ show a subset of results of 50 -min counts sequentially plotted against the observation number (we show the first and last 20 counts for illustration).

${ }^{1}$ Scottish Universities Research and Reactor Centre, East Kilbride G75 0QU Scotland

${ }^{2}$ Department of Statistics, University of Glasgow, Glasgow G12 8QW Scotland 
TABLE 1. Summary of Counting Experiments

\begin{tabular}{clcll}
\hline Group & Counter & $\begin{array}{c}\text { No. of } \\
\text { samples }\end{array}$ & $\begin{array}{l}\text { Sample } \\
\text { geometry }\end{array}$ & $\begin{array}{l}\text { No. of } \\
\text { results }\end{array}$ \\
\hline 1 & $2000 \mathrm{CA} / \mathrm{LL}$ & 4 & $2 \mathrm{~g} \mathrm{C}_{6} \mathrm{H}_{6}$ & 100 \\
2 & $2260 \mathrm{XL}$ & 4 & $2 \mathrm{~g} \mathrm{C}_{6} \mathrm{H}_{6}$ & 100 \\
3 & $2260 \mathrm{XL}$ & 4 & $0.5 \mathrm{~g} \mathrm{C}_{6} \mathrm{H}_{6}$ & 50 \\
\hline
\end{tabular}
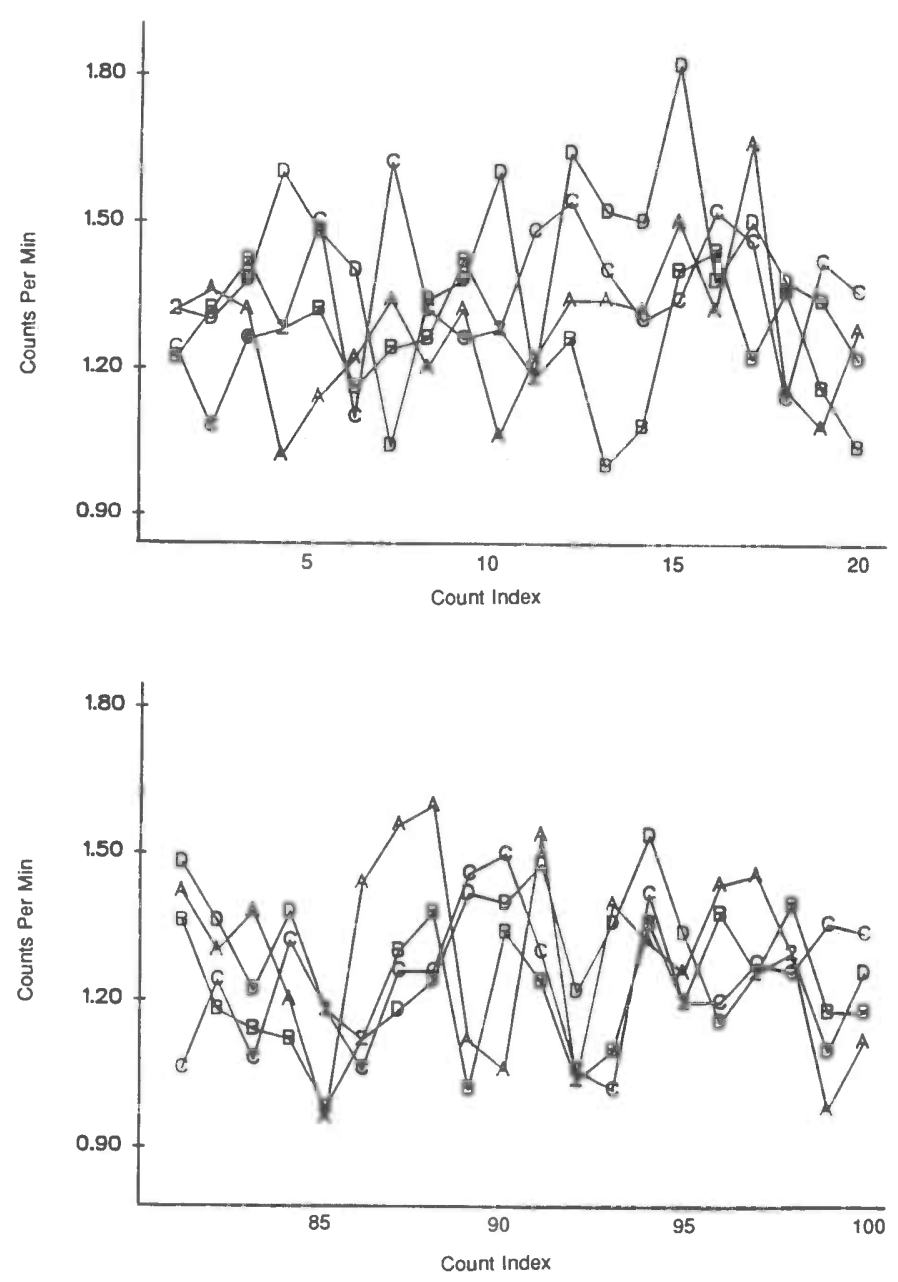

Fig. 1A. Time series plots for 4 backgrounds from $2000 \mathrm{CA} / \mathrm{LL}$ (first $20 \times 50$ min counting periods)

Fig. 1B. Time series plots for 4 backgrounds from $2000 \mathrm{CA} / \mathrm{LL}$ (last $20 \times 50$ min counting periods)

\section{THE APPLICABILITY OF POISSON COUNTING STATISTICS}

The precision of the counting experiment is frequently assumed to be governed by Poisson counting statistics; commonly, however, the Poisson counting statistic may yield only a lower limit. Currie (1972) writes that the proper evaluation of any excess 'random error' is important for detection of sources of instability and for estimation of realistic experimental precision. Detailed work on a high-precision liquid scintillation system (Pearson 1979) has indicated that additional sources of variability include benzene purity, vial reproducibility and the effect of atmospheric 
pressure on background. The basic assumption underlying Poisson counting statistics is that the variance is taken to be equal to the average of the counts observed; in other than counting experiments, the variance is typically estimated from deviations based on replicates. To evaluate the Poisson nature of each of the background samples in turn, we have considered the Poisson index of dispersion, P (Kotz, Johnston \& Read 1986), where

$$
P=\frac{\sum_{i=1}^{n}\left(x_{i}-\bar{x}\right)^{2}}{\bar{x}}
$$

where $x_{i}$ is the individual count and $\bar{x}$ is the overall mean, i.e.,

$$
\overline{\mathrm{x}}=\frac{\Sigma \mathrm{x}_{\mathrm{i}}}{\mathrm{n}} \text {. }
$$

If the data are distributed as a Poisson, then $\mathrm{P}$ will have an approximate chi-squared distribution. The index given in Equation (1) is an empirical form of the dispersion statistic given by Cox and Lewis (1966). The numerator is the sample variance; the denominator is the sample mean. Typically, we would conclude that the data are not Poisson-distributed if $\mathrm{P}$ exceeded a typical value, given in the $\chi^{2}(n-1)$ table. This would be interpreted as indicating overdispersion, i.e., more variability than expected. For the 12 samples considered in this experiment, only one did not exhibit Poisson properties. This may have occurred by chance or may reflect an effect of the vial itself or changes in the chemistry of the vial contents.

Figure 2 is a typical plot of the ratio of the Poisson error to the standard deviation about the mean for blocks of 10 counts in a single batch, where there is no evidence of extra Poisson variation. We show the line, ratio $=1$, and we see the scatter of points around this ideal value. An extreme observation is observed at subgroup 9 , which would then result in a re-examination of the original set of 10 counts.

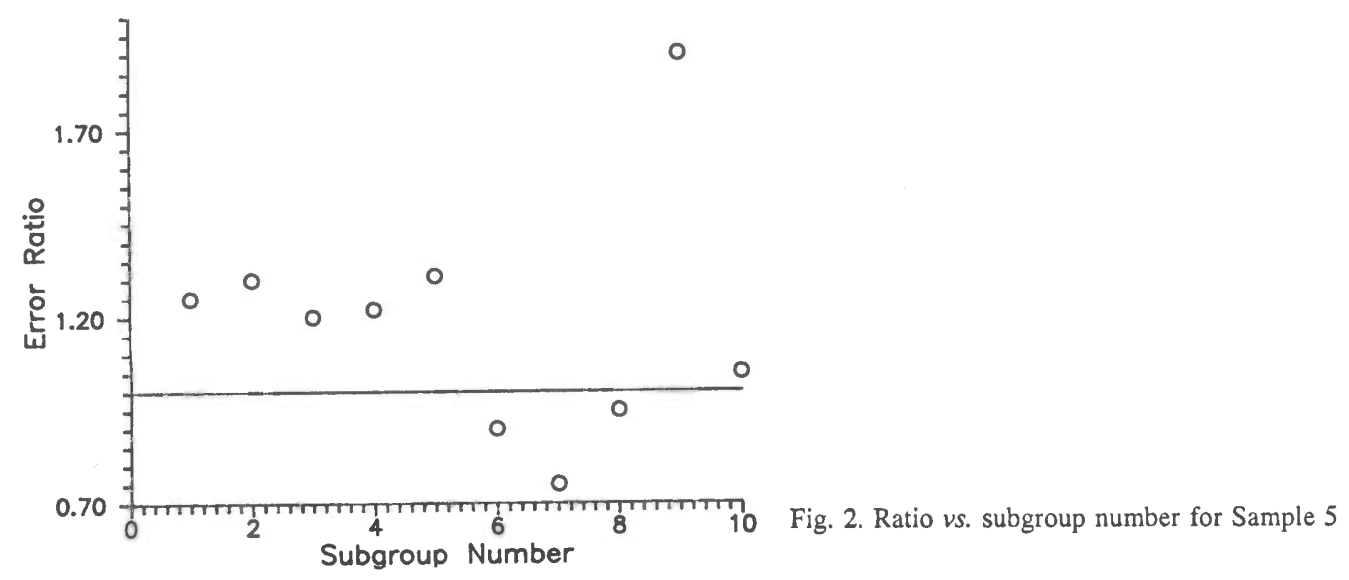

\section{STABILITY OF THE COUNTING SYSTEM}

As technology pushes background levels lower and lower, stability of the system must also be guaranteed. Random fluctuations, of approximately equal size to the expected count rates, cannot be tolerated, and sensitive, simple procedures are required to continually monitor system stability. The recently published Quality Assurance protocol (Long \& Kalin 1990) acknowledges the impor- 
tance of system instability, about which several papers have been published (Switsur 1990a, b). We discuss the techniques available and illustrate from other experiments their application to the counting data. Specifically, we discuss the detection of drift using the mean square successive difference (MSSD) method (Hooton \& Parsons 1973) and quality control charts.

\section{Quality Control Charts}

We use two control charts (Switsur 1990b), the $\bar{x}$ chart, to assess the process mean level, and the $\mathrm{s}$ chart, to assess the process variability. The charts are produced by defining a number of subgroups. In this instance, a sensible definition is linked to the quasi-simultaneous batch-counting technique, i.e., based on sequences of 10 counts, which were previously used in the assessment of the Poisson nature of the data.

For the s-chart, we plot the standard deviation of each subgroup against the subgroup number, superimpose a horizontal line representing the average standard deviation, and draw two lines of upper and lower control limits, defined below.

$$
\begin{aligned}
& \mathrm{LCL}=\frac{\bar{s}}{\mathrm{C}_{4}} \sqrt{\frac{\chi^{2}(\mathrm{n}-1,0.025)}{\mathrm{n}-1}} \\
& \mathrm{UCL}=\frac{\bar{s}}{\mathrm{C}_{4}} \sqrt{\frac{\chi^{2}(\mathrm{n}-1,0.975)}{\mathrm{n}-1}}
\end{aligned}
$$

$\mathrm{C}_{4}$ is tabulated (Ryan 1989).

Figure 3 shows a typical s chart, indicating that the process variability is in control, since none of the plotted (3) points lie outside the control limits.

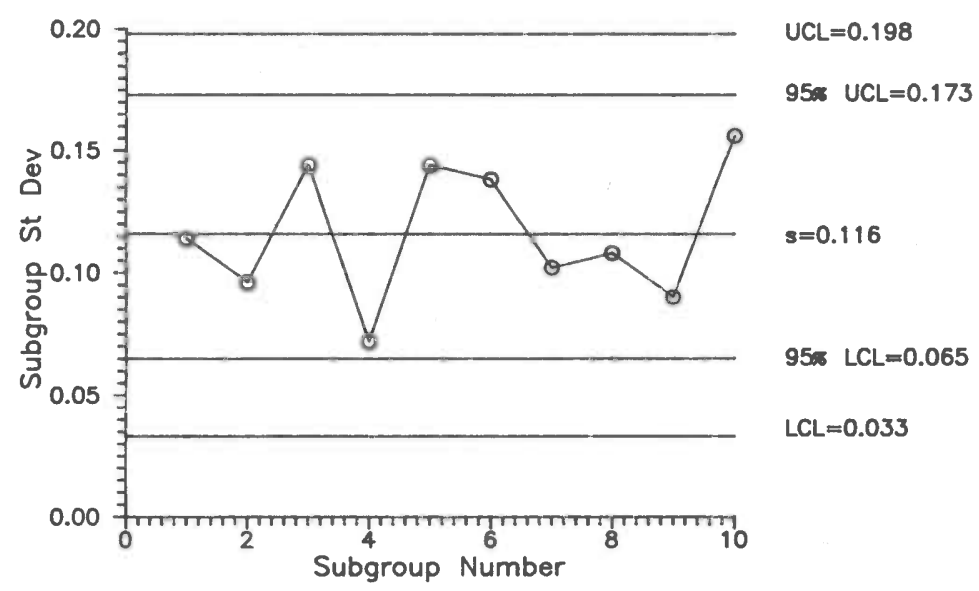

Fig. 3. S chart for Sample 8

The $\overline{\mathrm{X}}$ chart is constructed in a similar manner, with subgroup means now being plotted against subgroup number, the overall mean $\overline{\bar{x}}$, being superimposed, and control limits of $\overline{\bar{x}} \pm A_{3}$ s being plotted (again $A_{3}$ values are tabulated (Ryan 1989)). Figure 4 shows the $\bar{x}$ chart for the same sample as Figure 3. Again, the process mean appears in control. Overall, control charts were produced for all samples used in this experiment and indicate stable instrumentation. However, 


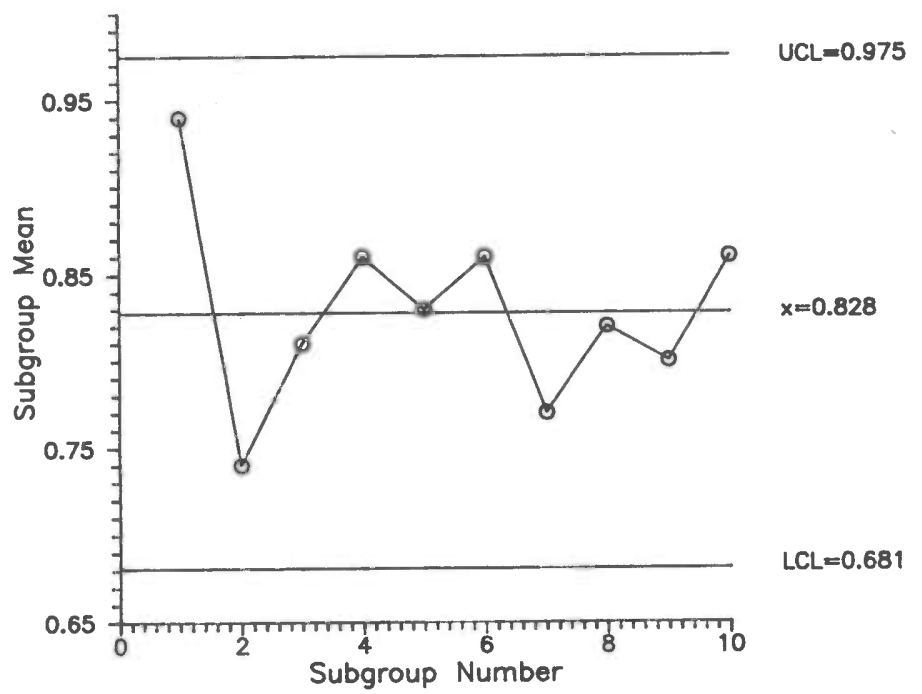

Fig. 4. $\bar{X}$ chart for Sample 8

these charts are intended to demonstrate substantial oscillations or large-scale drift, but any significant small-scale drift within the defined control limits may not be detectable.

\section{Detection of Drift}

The MSSD can be used to detect drift in a sequence of counts (Hooton \& Parsons 1973). This method takes account of the order of the measurements and is calculated from

$$
\begin{aligned}
& v^{2}=\frac{\sum_{i=1}^{n-1}\left(x_{i+1}-x_{i}\right)^{2}}{(n-1)} \\
& s^{2}=\frac{\sum_{i=1}^{n}\left(x_{i}-\bar{x}\right)^{2}}{(n-1)} .
\end{aligned}
$$

$v^{2}$ will be affected by rapid oscillations but less by gradual drift than $\mathrm{s}^{2}$. The statistic, $M=v^{2} / \mathrm{s}^{2}$, is used in the construction of $T$, where

$$
T=\frac{(n-1)(n+1)\left(1-\frac{M}{2}\right)}{(n-2)} .
$$

This follows a $t(n-1)$ distribution under the assumption of no drift. Although we found no significant deviations using the control charts, in several samples we did find evidence for significant small drifts in the results. Figure 5 shows an s chart demonstrating significant, small-scale drift, although no value exceeded the control limits. Consideration of all the control charts highlighted a small number showing such patterns. When combined, these techniques are then sensitive to substantial oscillations and small-scale drifts, and provide useful laboratory procedures for routine quality assurance checking. We interpret these diagrams to mean that there is instrumental stability with occasional particular features associated with specific samples. 


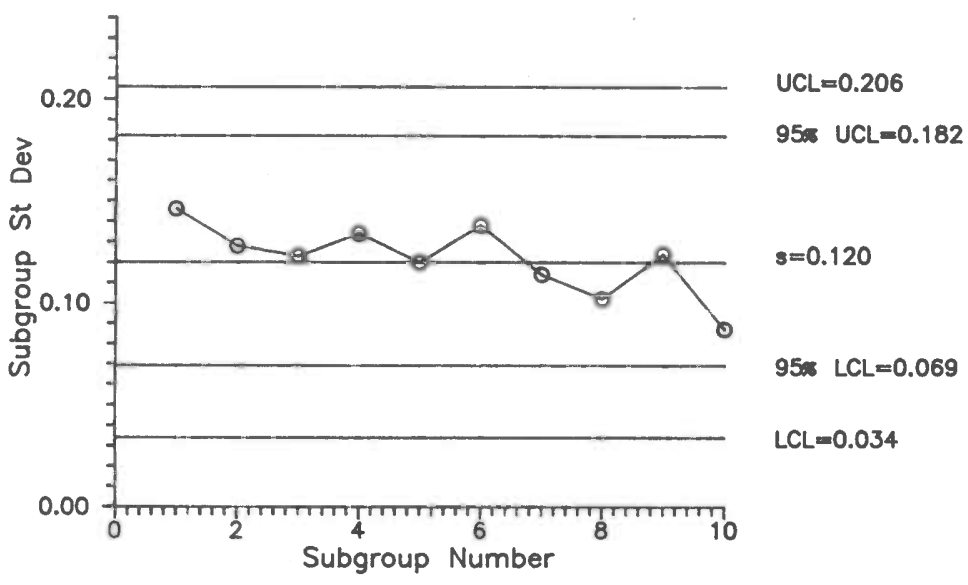

Fig. 5. S chart for Sample 6

\section{DISCUSSION AND CONCLUSIONS}

We have investigated long sequences of background counts collected to study the nature of the counting system. Of particular interest has been the appropriateness of Poisson statistics and the stability of the counting systems. Using the Poisson index of dispersion and ratio of error, we found no evidence that Poisson counting statistics are inappropriate.

Using both the $\bar{x}$ and s control charts, we found evidence of behavior inconsistent with a stable system in only one sample. For the question of small incremental drift, we used MSSD statistics, and, in several situations, found evidence of small drifts that would not be detected using control charts. However, these were not consistent within counting batches, and would tend to suggest that they are not instrument-related. This paper has provided illustrations of existing statistical techniques applied to ${ }^{14} \mathrm{C}$ counting data, which may be used routinely in laboratory quality assurance procedures.

\section{REFERENCES}

Cox, D. R. and Lewis, P. A. W. 1966 The Statistical Analysis of a Series of Events. London, Methuen Monograph.

Currie, L. A. 1972 The limit of precision in nuclear and analytical chemistry. Nuclear Instruments and Methods 100: 387-395.

Hooton, K. A. H. and Parsons, M. L. 1973 Equipment stability in $\mathrm{x}$-ray fluorescence spectrometry and radioactive counting - a statistical approach. Analytical Chemistry 45(13): 2218-2227.

Kotz, S., Johnston, N. L. and Read, C. B. 1986 Encyclopedia of Statistical Sciences, Vol. 7. New York, Wiley - Interscience.

Long, A. and Kalin, R. M. 1990 A suggested quality assurance protocol for radiocarbon dating laboratories. In Scott, E. M., Long, A. and Kra, R. S., eds., Proceedings of the International Workshop on Intercomparison of ${ }^{14} \mathrm{C}$ Laboratories. Radiocarbon 32(3): 329-334.

Noakes, J. E. and Valenta, R. J. 1989 Low background liquid scintillation counting using an active sample holder and pulse discrimination electronics. In Long, A. and Kra, R. S., eds., Proceedings of the 13th International ${ }^{14} \mathrm{C}$ Conference. Radiocarbon 31(3): 332341.

Pearson, G. W. 1979 Precise ${ }^{14} \mathrm{C}$ measurements by liquid scintillation counting. Radiocarbon 21(1): 121.

Ryan, T. P. 1989 Statistical Methods for Quality Im. provement. New York, John Wiley \& Sons Inc.

Switsur, R. 1990a A consideration of some basic ideas for quality assurance in radiocarbon dating. In Scott, E. M., Long, A. and Kra, R. S., eds., Proceedings of the International Workshop on Intercomparison of ${ }^{14} \mathrm{C}$ Laboratories. Radiocarbon 32(3): 342-346.

1990 b Statistical quality control graphs in radiocarbon dating. In Scott, E. M., Long, A. and Kra, R. S., eds., Proceedings of the International Workshop on Intercomparison of ${ }^{14} \mathrm{C}$ Laboratories. Radiocarbon 32(3): 347-354. 